\title{
It's Not All in Our Genes!
}

The genetics revolution, through which we are living, is in the midst of changing the way we understand the biology that underlies complex behavioral disorders. By virtue of this new technology, we have been catapulted into an age of molecular medicine that is defining the very way we look at disease. In the case of diseases of the brain and complex behavior, however, the dualistic concept of environment and genes should become as anachronistic as the Descartian concept of somapsyche. For the brain, there is always a dynamic interaction with the environment. No behavior is manifest in isolation of the context, and this environment will always shape the distinct form of its expression. On the other hand, the brain is altered directly and indirectly by the perceptual input derived from the setting and the consequent response of the individual. Given the dynamic properties of the neuroplastic processes in the brain, repeated exposure to adverse circumstances might be expected to alter subsequent function. Such alteration in the physiology of the brain can result in both enhancement and decrement in the functional output of individual neuronal pathways. Thus, it is time we begin to account for the accumulated impact of the environment over an extended period of time in formulating concepts regarding chronic disease models involving soma and psyche.

In this month's Perspective article, Dr. McEwen sets forth the concept of "allostasis" and "allostatic loading" as it relates to exposure to stressful events over a lifetime. This concept is not unfamiliar when it comes to the accumulated effects of exposure to toxins, smoking, and high fat diets on medical morbidity in both individuals and subpopulations. Similarly, we have long recognized that stressful events, e.g., battlefield, concentration camps, terrorist bombings, etc., can adversely impact coping responses of individuals at both psychological and sociological levels. Dr. McEwen highlights the application of allostasis to biological changes in the brain that may not only set the stage for maladaptive physiological responses in an individual over time, but may also lay the foundation for the demographics of medical risk assessment for subpopulations of individuals subject to accumulated periods of exposure to a variety of environmental stressors during both development and adulthood.

While the concept of allostasis must be better understood at the level of brain biology, our experimental paradigms would do well to be carried out in the context of the differences in genetic background. Ultimately, it is this dynamic between genetic background and allostasis that will drive the expression of the medical illnesses and complex behavioral disorders in question. Most recently, Kendler et al. (1999) have determined that stressful life events certainly play a significant causal role in the precipitation of depression, but individuals shown to be at risk for depression also appear to segregate preferentially into high risk environments. Thus, we return to the dynamic properties of the gene-environment dyad, wherein the concept of allostasis as presented by Dr. McEwen may be particularly useful in fully understanding the patterns of clinical expression of both medical and psychiatric diseases across sectors of our population, as well as cultures throughout the world. As both basic and clinical neuroscientists we would do well to keep this in mind.

Robert H. Lenox, M.D. Editor-In-Chief

\section{REFERENCES}

McEwen BS (1999): Allostasis and allostatic load. Implications for neuropsychopharmacology. Neuropsychopharmacology 22:108-124

Kendler KS, Karkowski LM, Prescott CA (1999): Causal relationship between stressed life events and the onset of major depression. Am J Psychiatry 156:837-841 